\title{
Dorsolateral Prefrontal Cortex Activity and Neuromodulation in Crack-Cocaine Dependents during Early Abstinence
}

Catarine Lima Contia*, Janine Andrade Moscona, Ester Miyuki and Nakamura-Palaciosa

Laboratory of Cognitive Sciences and Neuropsychopharmacology, Program of Post-Graduation in Physiological Sciences, Federal University of Espírito Santo, VitóriaES, Brazil

"Corresponding author: Catarine Lima Conti, Programa de Pós-Graduação em Ciências Fisiológicas, Centro de Ciências da Saúde, Universidade Federal do Espírito Santo, Av. Marechal Campos, 1468, 29.047105 Vitória, ES, Brazil, Tel: +55-27-3335-7337; Fax: + 55(27) 3335-7330; E-mail: catarineconti@hotmail.com

Received date: April 22, 2016; Accepted date: May 23, 2016; Published date: May 30, 2016

Copyright: $\odot 2016$ Contia CL, et al. This is an open-access article distributed under the terms of the Creative Commons Attribution License, which permits unrestricted use, distribution, and reproduction in any medium, provided the original author and source are credited.

\begin{abstract}
Rationale: It is broadly known that addicted subjects have frontal dysfunction which is translated into: 1) low frontal activity in areas related to executive functions involved in the control over drug use; and 2) high frontal activity in areas related to incentive salience involved in the craving for drug use.

Method: Electrical activity was correlated with days of abstinence and then sham-controlled session of transcranial direct current stimulation (tDCS) was performed in crack-cocaine users up to one month of abstinence. They were randomly divided to receive left cathodal/right anodal tDCS (20 min, $\left.2 \mathrm{~mA}, 35 \mathrm{~cm}^{2}\right)$ or sham procedure over the dorsolateral prefrontal cortex (DLPFC).
\end{abstract}

Results: Absolute values from low-resolution brain electromagnetic tomography (LORETA) indicated that the activity in both left and right DLPFC increases progressively from the tenth day of abstinence. The left cathodal/right anodal tDCS prevented the increase of the activity in the left DLPFC facing crack-cocaine cue presentation in the visual P3 time window (350-600 ms). Spontaneous changes in the right DLPFC were not affected by tDCS.

Conclusion: Prefrontal tDCS seems to modulate the DLPFC response to drug cue exposure in this preliminary study with crack-cocaine users suggesting this technique as an effective tool in reducing craving during early abstinence.

Keywords: Crack-cocaine dependent; Dorsolateral prefrontal cortex; Event-related potential; tDCS

\section{Introduction}

The mesocorticolimbic dopamine system and the nigrostriatal dopamine system both contribute to cue-induced cocaine seeking [1-3] and other behavioral effects of cocaine, including cocaine reward $[4,5]$. It is well known that areas from prefrontal cortex (PFC) that are involved in these drug-related processes are activated when drug addicted are exposed to either the drug or some drug-cue [6-14] postulated that this enhanced activity of PFC could contribute to the compulsive self-administration and the lack of control (impaired inhibition) in addicted subjects and also contribute to disruptive cognitive operations that impair judgment and favor relapse [14].

Experimental investigations of addictive phenomena by using a cuereactivity paradigm have been performed extensively [15-18] and a growing amount of evidence suggests that electroencephalographic activity of frontal-central areas increases when cocaine users are exposed to pictures of cocaine compared to neutral images [19-21]. Using scalp surface electrodes, the event-related potential (ERP) can be recorded in response to a variety of sensory stimuli, and much information can be obtained from these electrodes after a given task with a high temporal resolution.

One of the most studied endogenous ERP components is the P3 wave, which is the largest positive going peak occurring within a time window of $250-500 \mathrm{~ms}$. The P3 wave is typically observed in more anterior brain areas [22] and is sensitive to general and specific arousal, contributing to attention activation and information processing [23]. The association between P3 amplitude and cue-reactivity has been described in volunteers with history of cocaine use $[8,24]$ and other drugs $[25,26]$. These studies report increased craving after the presentation of drug-related cues, as well as an increased P3 amplitude.

The tDCS is a technically simple tool of non-invasive brain stimulation used to modulate neuronal resting membrane potential in humans leading to changes of cortical excitability [27-29] and it is well established that cathodal current decreases cortical excitability while anodal current increases excitability [30-33].

Our hypothesis is that tDCS over DLPFC (applied just before cuereactivity paradigm) is able to change the cortical activity in this area during the cognitive potential P3 while crack-cocaine users are visualizing drug cues.

So, the objective here is to unprecedentedly investigate the neurophysiological effect of tDCS on the brain of crack-cocaine addicted patients through the cue-reactivity paradigm to check if neuromodulation is able to change brain activity while they are visualizing drug cues.

These are preliminary studies in order to cautiously advance with the proposal of using tDCS in the treatment of crack-cocaine dependence. 


\section{Material and Methods}

\section{Subjects}

This study included sixteen crack-cocaine addicted subjects as defined by the DSM-IV, recruited from the Center for Psychosocial Care for treatment of abuse and dependence of psychoactive substances disorders in Espírito Santo, Brazil. All participants had normal or corrected-to-normal vision.

The inclusion criteria for this study were: (1) patients between the age of 18 and 60 years; (2) met criteria for crack-cocaine dependence according to the Diagnostic and Statistical Manual of Mental Disorders, fourth edition (DSM-IV), as determined by clinic evaluation; (3) no more than 30 days of abstinence; (4) in stable clinical condition with no need for inpatient care; and (5) able to read, write, and speak Portuguese. Conversely, exclusion criteria included: (1) a diagnosis of epilepsy, convulsions, or history of severe brain injury; (2) any contraindication for electrical brain stimulation procedures such as electronic implants or metal implants; (3) suspected pregnancy for female participants; and (4) cardiac pacemaker.

Treatment and data collection were conducted according to the ethical principles in the Declaration of Helsinki, which are equivalent to those established by the Ethics Committee for Research at the Center of Health Sciences, Federal University of Espírito Santo, Brazil where this study was conducted. Ethical approval for this study was provided by the Brazilian Institutional Review Board of the Federal University of Espírito Santo (registration 296/10), Brazil. We are presenting results from the study registered at the ClinicalTrials.gov Protocol Registration System under identifier NCT01337297.

\section{General Procedures}

The sixteen subjects enrolled in this study were divided into two groups that received two types of electrode placement: 1) six subjects received left anodal tDCS; 2) ten subjects received left cathodal/right anodal tDCS. The protocol of left anodal tDCS had to be interrupted because all patients that were receiving the real procedure were relapsing. At this point, we changed the electrode placement and continued the trial. At this manner, the results from anodal tDCS were not considered because of the very small number. This observation also reinforces the need of cautiously including a limited number of patients in these preliminary studies of the use of neuromodulation in addicted subjects.

They were fully informed about the experimental protocol and voluntarily signed an informed consent form and randomly assigned to receive real brain stimulation (tDCS group) or receive a simulation of this procedure (sham-tDCS group). The experimental protocol consisted of global physical and clinical examination and electrophysiological recording of brain activity during the random visual presentation of three drug-related images and three neutral images before and after a single session of $\mathrm{tDCS}$.

\section{EEG recording}

The electrophysiological recording was obtained through a 32channel system (QuickAmp40, BrainProducts Ltd., Munich, Germany) using active electrodes with an integrated impedance converter for noise subtraction circuits (actiCAP BP; BrainProducts Ltd, Munich, Germany) placed on the scalp following the International 10/20 system. Data were recorded with a sampling rate of $1000 \mathrm{~Hz}$, analog filtered between 0.016 and $1000 \mathrm{~Hz}$ with common average reference.

\section{Experimental design and task}

We adapted a cue-reactivity paradigm [34] following standard cue reactivity paradigms well established for pictures [35] and videos [36]. During the pictures presentation the subjects were asked to press a button whenever the crack-related pictures were presented, and to withhold the response (50\%) when the neutral pictures were presented. This allowed us to assert that patients were aware about pictures presentation and the number of errors (false or miss) of the subjects included in this study was zero or 1 .

\section{Stimuli}

Three pictures related to the consumption of crack-cocaine (i.e., crack-related cues) such as crack rocks, pipes or paraphernalia used for substance use, and someone inhaling the substance constituted the target visual stimuli. Meanwhile, three neutral pictures that were unrelated to the consumption of crack (i.e., neutral cues) such as a landscape with a small road, a field of flowers, and a butterfly constituted the non-target visual stimuli.

The trial consisted by the running of a randomized sequence of 90 visual presentations (15 times for each picture) approximately at eye level, using a 17 inch monitor $(1280 \times 1024 \times 32$-bit color, $60 \mathrm{~Hz}$ refresh rate). Each picture was presented for $1000 \mathrm{~ms}$, at intervals of $2000 \mathrm{~ms}$, so the entire procedure lasted $4.5 \mathrm{~min}$. The default screen consisted of a black background at all times. All pictures were presented using Presentation 10.0 software (Presentation, Neurobehavioral Systems, Inc, Albany, CA).

\section{Data processing}

All EEG data were processed using BrainVision Analyzer 2.0 Professional (BrainProducts Ltd., Munich, Germany). Data were offline filtered from $1 \mathrm{~Hz}$ to $10 \mathrm{~Hz}$ and after ocular correction by independent component analysis and visual inspection for artifact removal all datasets were segmented into epochs from -200 to $800 \mathrm{~ms}$ relative to picture onset and averaged. All epochs were retained. Baseline correction was performed using the prestimulus interval (i.e., -200 to $0 \mathrm{~ms}$ ). Low-resolution brain electromagnetic tomography (LORETA) was used to estimate the three-dimensional intracerebral current density distribution $\left(\mu \mathrm{A} / \mathrm{mm}^{2}\right)$. Together with the high temporal resolution of ERP, these functional images of electric neuronal activity have been exploited successfully in applications that validated LORETA with Magnetic Resonance Imaging (MRI) and Positron Emission Tomography (PET) findings [37-40]. In this study we focused our analysis on DLPFC as the region of interest (ROI) and the mean of LORETA absolute value from $350 \mathrm{~ms}$ to $600 \mathrm{~ms}$ (P3) from this ROI was plotted. Middle frontal gyrus including Brodmann areas 9 and 46 was the evaluated area accurately during P3 segment.

\section{Brain stimulation}

Direct currents were transferred via a pair of carbonated-silicone electrodes $\left(35 \mathrm{~cm}^{2}\right)$ with a thick layer of high conductive gel for EEG underneath them according to our previous study [41]. The electric current was delivered by an electric stimulator (Striat, Ibramed Indústria Brasileira de Equipamentos Médicos Ltd, São Paulo, Brazil). For brain stimulation, the cathode electrode was placed over F3 (left 
DLPFC) and anode over F4 (right DLPFC) according to the 10-20 international system.

The currents flowed continuously for $20 \mathrm{~min}$ with an intensity of 2.0 $\mathrm{mA}$. For the sham procedure, the electrodes were placed in the same position, but the stimulator was turned off after $20 \mathrm{~s}$ (gradually from 2 $\mathrm{mA}$ to $1 \mathrm{~mA}$ and then was off) in such a way that subjects felt the initial itching sensation at the beginning, but received no current for the rest of the stimulation period. This procedure allowed keeping subjects blind for the respective stimulation condition [42].

\section{Statistical analyses}

Data were presented by mean and standard deviation (S.D.). Once data from DLPFC activity in line with days of abstinence were normally distributed (D'Agostino-Pearson omnibus normality test) linear regression was performed and $\mathrm{P}$ value was according to Pearson correlation. The effect of tDCS was analyzed with the Wilcoxon test, since the differences in activity between before and after tDCS did not fulfill the criteria for normality. A p-value of 0.05 or less was considered to indicate a statistical significance. GraphPad Prism 6.0 (GraphPad Software Inc, USA) was employed for statistical analysis and graphic presentations.

\section{Results}

\section{Sample}

Ten crack-cocaine dependents that had no more than 30 days of abstinence were included in these preliminary findings. Concomitant use of other drugs of abuse includes alcohol, nicotine, marijuana and cocaine. Comorbidities present were mood disorder (depression), generalized anxiety disorder and bipolar disorder. The standard of crack use, considering the number of rocks per week, varied from 5 to 50. The list of medication included in this study, their classes and doses are presented in the table below (Table 1).

\begin{tabular}{|l|c|c|}
\hline Drugs & Class & Dose (mg/day) \\
\hline Amitriptyline & TCS & $25-50$ \\
\hline Chlorpromazine & Antipsychotic & 100 \\
\hline Promethazine & Antipsychotic & 25 \\
\hline Diazepam & Benzodiazepine & 20 \\
\hline Buspirone & Anxiolytic & 10 \\
\hline Sertraline & SSRI & 50 \\
\hline Risperidone & Antipsychotic & 4 \\
\hline
\end{tabular}

Table 1: List of medication, class and dose from patients included in this study.

\section{DLPFC activity from early abstinent crack-cocaine users}

The cortical activity was measured during the visual cue-reactivity paradigm. The points at the graphic (Figure 1) correspond to the mean of absolute value from DLPFC LORETA from 350 to $600 \mathrm{~ms}$ relative to picture onset indicating the activity of DLPFC during the P3 brain cognitive potential while subjects were visualizing crack-related pictures.

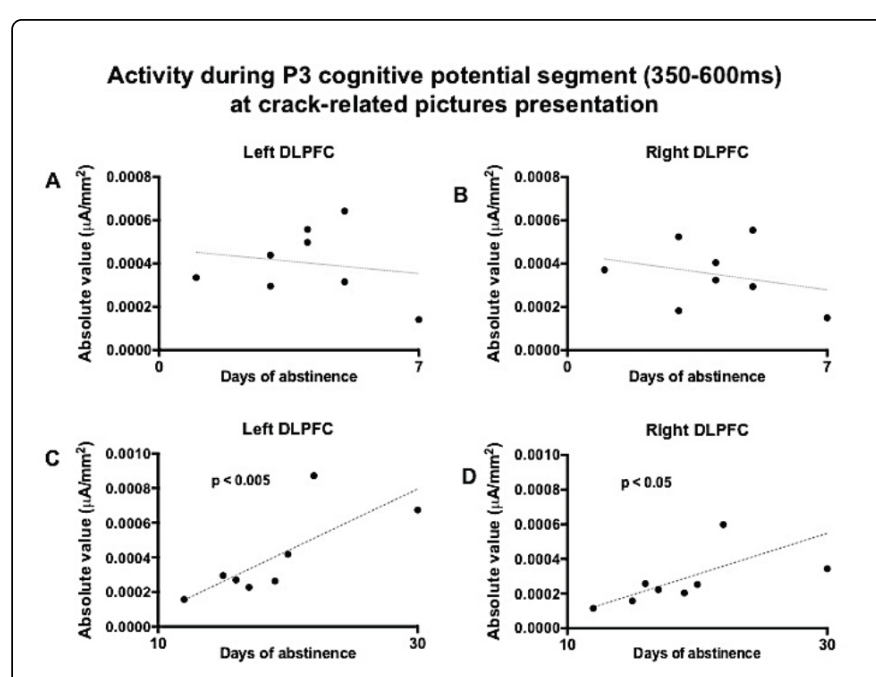

Figure 1: Current density distribution (LORETA) and correlations with days of abstinence in crack-cocaine addicted individuals. The plots show the association between absolute values compared to days of abstinence in the both left and right DLPFC during P3 (350-600 ms), and the corresponding linear regression line (significant result for C: $\mathrm{r}=0.70, \mathrm{p}<0.005$ and $\mathrm{D}: \mathrm{r}=0.59, \mathrm{p}<0.05$ ).

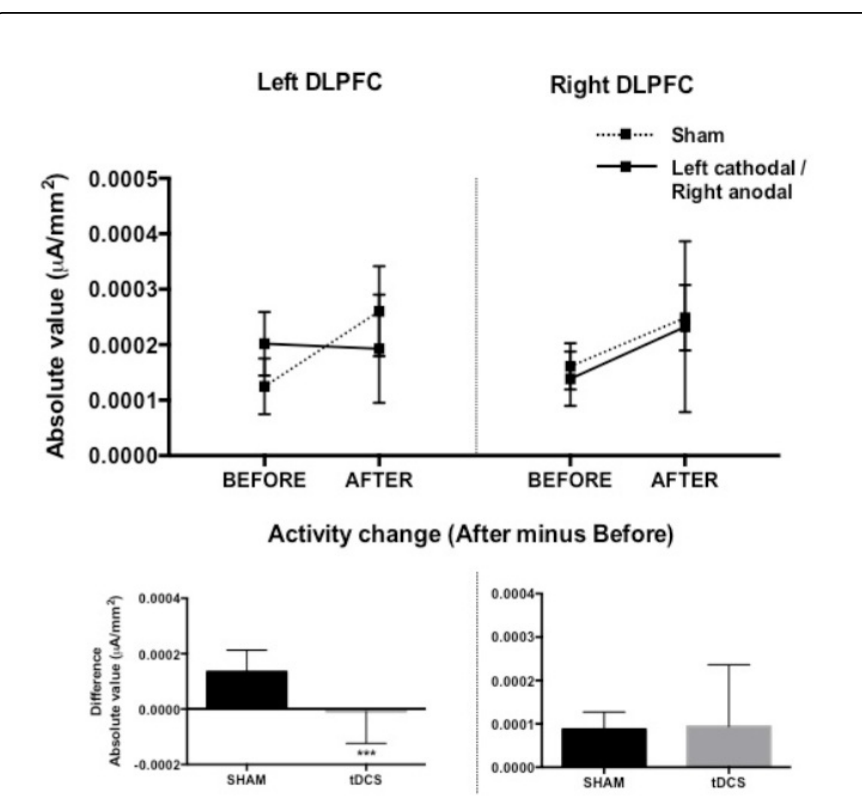

Figure 2: Current density distribution (LORETA) responses in the left and right DLPFC under visual exposition to drug-related cues and bar representation of after minus before activity in the tDCS on sham and real group (single session of sham/tDCS, $20 \mathrm{~min}, 2 \mathrm{~mA}$, $35 \mathrm{~cm}^{2} ;{ }^{* * *} \mathrm{p}<0.0001$; Wilcoxon non-parametric paired test. All data are represented by mean $\pm \mathrm{SD}$ ).

Up to seven days, the DLPFC activity had no correlation with time of abstinence. However, the significant effect of this early abstinence in the DLPFC activity starts to appear from the tenth day, from when the increase of activity is correlated with days of abstinence (D'Agostino 
and Pearson omnibus normality test followed by linear regression $\mathrm{p}=0.0045$ for left DLPFC and $\mathrm{p}=0.014$ for right DLPFC, Pearson correlation coefficients were computed).

\section{ERP before and after tDCS}

Subjects received real or sham tDCS. No significant difference was found between them related to the days of abstinence (sham $=15.25 \pm$ $11.66, \mathrm{n}=5 ; \mathrm{tDCS}=10.22 \pm 7.22, \mathrm{n}=5 ; \mathrm{p}=0.2959$, unpaired $\mathrm{t}$-test).

Absolute values of DLPFC activity were analyzed before and after brain stimulation (Figure 2). The tDCS was able to prevent the increasing P3 brain activity on the left DLPFC when patients were visualizing drug cues $(\mathrm{p}<0.0001$; Wilcoxon nonparametric paired test).

\section{Discussion}

In this study we observed that the brain activity measured precisely during P3 cognitive potential from both left and right DLPFC of crackcocaine users increases from the tenth day of abstinence. Hereafter, we observed that a single session of left cathodal/right anodal tDCS over DLPFC prevented the increase of the brain activity in the left DLPFC while patients were visualizing crack-related pictures. No difference was observed in the right DLPFC.

A great challenge of studying neuromodulation is the variety of factors associated with drug dependence, as comorbidities - generally mood and anxiety disorders, concomitant drug use and different classes of medication used for the treatment of those comorbidities. The standardization of the treatment for each drug abuse dependent will be benefited by trials with lager samples and our team has been working in this sense. Far as we know, we are the first group purposing neuromodulation in the treatment of crack-cocaine dependence and the aspects that strengthen the use of tDCS is its described effect on cognitive function. Cognitive impairment is a common mechanism in drug abuse, which involves the prefrontal cortex function. So, the focus of prefrontal cortex neuromodulation as an alternative treatment of crack-cocaine dependence is to increase the cognitive function allowing better control over drug use as discussed below. Following this rationale, cortical neuromodulation can be also purposed for the treatment of other drugs of abuse as we have suggested [43]

The PFC activity increases when patients are facing the drug or some drug-related cues. This evidence suggests that the PFC, at this condition, may be much more involved in the craving response rather than in the control over it. It was already showed that the metabolism in the PFC of cocaine-addicted subjects exposed to a stimulant drug is increased (an effect that was associated with craving for the administered drug) while non-addicted subjects exposed to the same drug had decreased metabolism in these regions. Thus, the activation of these prefrontal regions with drug exposure may be specific to addiction and associated with the enhanced desire for the drug [44]. Furthermore, the enhancement of activity in drug-related processes including emotional responses (medial OFC and ventromedial PFC in craving), automatic behaviors (OFC in drug expectation and ACC in attention bias) and also higher-order executive responses through the activation of the DLPFC involved in drug-related working memories [44] may constitute the prominent factor that precedes relapse.

While short-term abstinence was proposed to increase cue-induced response in the PFC, it was proposed that cognitive intervention attenuates this response [45]. When cocaine abusers purposefully inhibit craving when exposed to conditioned drug-cues, specific changes in brain regions that process reward and prediction of reward occur, or else, regions involved in processing conditioned responses decrease their activities. According to Volkow et al. [46] "The frontal mediation of a neural circuit involved in the craving response provides a target for topdown cognitive interventions that may be therapeutically beneficial. Interventions that strengthen a weakened but still functional fronto-accumbal circuit may increase the ability of cocaine abusers to block or reduce the drug craving response." Following this rationale, we are now moving toward the investigation of the effects of cathodal tDCS modulation of the left DLPFC once we observed in this study that cathodal tDCS over the left DLPFC decreased the DLPFC activity during crack-cue visualization.

Experimental studies have shown that exposure to drug-related stimuli increases cravings in drug-dependent individuals. However, the most commonly used measures are subjective self-reports, which assess the 'desire' for a particular substance though objective physiological and behavioral responses can also be measured. ERPs studies have been increasingly used for a more objective measurement of the motivational attribute of drug-related stimuli in human subjects. In spite of its low spatial resolution, ERPs have an excellent temporal resolution, allowing the investigation of the time course of emotional processing and drug cue reactivity. We enforce this tool as an objective outcome for research in the cognitive field considering that ERPs capture different stages within emotional processing and other components can be evaluated. An elegant study conducted by Dunning et al. found that deficient electrophysiological response to pleasant and unpleasant images was most evident during later processing in current users of cocaine; still according to these authors, this deficit may be indicative of impairments in sustaining non-drug-related goaloriented motivation, predisposing addicted individuals to drug use as a mechanism compensating for the reduced response to other reinforcement (including reward) [20].

Transcranial electric activity has been shown to be associated with frontal-related cognitive changes in healthy subjects and several psychiatric conditions [47-55]. Previous study from our lab showed specific clinical and electrophysiological (as indexed by P3) effects of tDCS on patients with alcohol dependence in which we demonstrated that anodal tDCS over left DLPFC resulted in an improved cognitive function [41]. Not only transcranial, but also epidural stimulation was already used to study emotion regulation and the impact of cognitive control on neural response to visual stimuli. Hajcak et al. [56] studied five patients with treatment-resistant mood disorders stereotactically implanted with stimulating paddles over DLPFC bilaterally and this study corroborated the role of DLPCF in regulating measures of neural activity that have been linked to emotional arousal and attention. Though the number of studies on frontal neuromodulation has been growing in psychiatric disorders, efforts are needed to propose this technique as an effective repetitive therapy in the treatment of such conditions.

Other issues that must be considered here are the possible mechanisms involved in the process of drug dependence development. There are evidences that neuroinflammation plays a role in the pathophysiology of addiction. Inflammatory cytokines are able to increase expression and activity of some neutrotransmitters, as dopamine and serotonin, or their receptors or any protein involved in their presence and activity in the synaptic cleft [57]. These neurotransmitters can induce a psychostimulant effect, which can mimics brain reward induced by drug administration. Additionally, drug abuse is involved in the up-regulation of inflammatory mediators 
[58]. These findings show that the pathways involved in the neuromodulation as treatment for addiction can be broadly explored.

There are limitations of this study that need to be considered. Samples of crack cocaine users were small because of their own clinical characteristics (high rates of drop-outs, low adherence to biopsychosocial or to any other therapeutics, etc.) and the complexity of the experimental protocol (clinical and cognitive evaluation, EEG/ ERP, tDCS), limiting assumptions about the clinical relevance of our findings. However, all data have been supported by the most cited literature in drug addiction.

\section{Conclusion}

Our findings show that crack-cocaine users during early abstinenceespecially from the tenth day-have a growing increase in the DLPFC activity during P3 cognitive potential $(350-600 \mathrm{~ms})$ while they are visualizing drug-cue. Hereafter, we observed that prefrontal tDCS modulates the DLPFC response to drug cue exposure. These data encourage additional studies on the cortical neuromodulation as an alternative tool in the treatment of drug addiction.

\section{References}

1. Belin D, Jonkman S, Dickinson A, Robbins TW, Everitt BJ (2009) Parallel and interactive learning processes within the basal ganglia: Relevance for the understanding of addiction. Behav Brain Res 199: 89-102.

2. Feltenstein MW, See RE (2008) The neurocircuitry of addiction: An overview. Br J Pharmacol 154: 261-274.

3. Shalev U, Grimm JW, Shaham Y (2002) Neurobiology of relapse to heroin and cocaine seeking: A review. Pharmacol Rev 54: 1-42.

4. Pierce RC, Kumaresan V (2006) The mesolimbic dopamine system: The final common pathway for the reinforcing effect of drugs of abuse? Neurosci Biobehav Rev 30: 215-238.

5. Wise RA (2009) Roles for nigrostriatal--not just mesocorticolimbic-dopamine in reward and addiction. Trends Neurosci 32: 517-524

6. Daglish MR, Nutt DJ (2003) Brain imaging studies in human addicts. Eur Neuropsychopharmacol 13: 453-458.

7. Garavan H, Pankiewicz J, Bloom A, Cho JK, Sperry L, et al. (2000) Cueinduced cocaine craving: Neuroanatomical specificity for drug users and drug stimuli. Am J Psychiat 157: 1789-1798.

8. Grant S, London ED, Newlin DB, Villemagne VL, Liu X, et al. (1996) Activation of memory circuits during cue elicited cocaine craving. Proc Natl Acad Sci U S A 93: 12040-12045.

9. McBride D, Barrett SP, Kelly JT, Aw A, Dagher A (2006) Effects of expectancy and abstinence on the neural response to smoking cues in cigarette smokers: An fMRI study. Neuropsychopharmacology 31: 2728-2738.

10. Sell LA, Morris JS, Bearn J, Frackowiak RS, Friston KJ, et al. (2000) Neural responses associated with cue evoked emotional states and heroin in opiate addicts. Drug Alcohol Depend 60: 207-216.

11. Tapert SF, Cheung EH, Brown GG, Frank LR, Paulus MP, et al. (2003) Neural response to alcohol stimuli in adolescents with alcohol use disorder. Arch Gen Psychiatry 60: 727-735.

12. Volkow ND, Wang GJ, Fowler JS, Hitzemann R, Angrist B, et al. (1999) Association of methylphenidate-induced craving with changes in right striato-orbitofrontal metabolism in cocaine abusers: Implications in addiction. The American journal of psychiatry 156: 19-26.

13. Wilson SJ, Sayette MA, Fiez JA (2004) Prefrontal responses to drug cues: A neurocognitive analysis. Nat Neurosci 7: 211-214.

14. Volkow ND, Fowler JS, Wang GJ, Goldstein RZ (2002) Role of dopamine, the frontal cortex and memory circuits in drug addiction: Insight from imaging studies. Neurobiol Learn Mem 78: 610-624.
15. Carter BL, Tiffany ST (1999) Meta-analysis of cue-reactivity in addiction research. Addiction 94: 327-340.

16. Hester R, Dixon V, Garavan H (2006) A consistent attentional bias for drug-related material in active cocaine users across word and picture versions of the emotional Stroop task. Drug Alcohol Depend 81: 251-257.

17. Modesto-Lowe V, Kranzler HR (1999) Using cue reactivity to evaluate medications for treatment of cocaine dependence: A critical review. Addiction 94: 1639-1651.

18. Sokhadze E, Singh S, Stewart C, Hollifield M, El-Baz A, et al. (2008) Attentional bias to drug- and stress-related pictorial cues in cocaine addiction comorbid with PTSD. J Neurother 12: 205-225.

19. Bauer LO, Kranzler HR (1994) Electroencephalographic activity and mood in cocaine-dependent outpatients: Effects of cocaine cue exposure. Biological psychiatry 36:189-197.

20. Dunning JP, Parvaz MA, Hajcak G, Maloney T, Alia-Klein N, et al. (2011) Motivated attention to cocaine and emotional cues in abstinent and current cocaine users--an ERP study. Eur J Neurosci 33: 1716-1723.

21. van de Laar MC, Licht R, Franken IH, Hendriks VM (2004) Event-related potentials indicate motivational relevance of cocaine cues in abstinent cocaine addicts. Psychopharmacology (Berl) 177: 121-129.

22. Katayama J, Polich J (1998) Stimulus context determines P3a and P3b. Psychophysiology 35: 23-33.

23. Polich J (2007) Updating P300: An integrative theory of P3a and P3b. Clin Neurophysiol 118: 2128-2148.

24. Franken IH, Hulstijn KP, Stam CJ, Hendriks VM, van den Brink W (2004) Two new neurophysiological indices of cocaine craving: Evoked brain potentials and cue modulated startle reflex. J Psychopharmacol 18: 544-552.

25. Littel M, Franken IH (2007) The effects of prolonged abstinence on the processing of smoking cues: An ERP study among smokers, ex-smokers and never-smokers. J Psychopharmacol 21: 873-882.

26. Namkoong K, Lee E, Lee CH, Lee BO, An SK (2004) Increased P3 amplitudes induced by alcohol-related pictures in patients with alcohol dependence. Alcohol Clin Exp Res 28: 1317-1323.

27. Nitsche MA, Cohen LG, Wassermann EM, Priori A, Lang N, et al. (2008) Transcranial direct current stimulation: State of the art 2008. Brain Stimul 1: 206-223.

28. Nitsche MA, Fricke K, Henschke U, Schlitterlau A, Liebetanz D, et al. (2003a) Pharmacological modulation of cortical excitability shifts induced by transcranial direct current stimulation in humans. J Physiol 553: 293-301.

29. Nitsche MA, Liebetanz D, Antal A, Lang N, Tergau F, et al. (2003b) Modulation of cortical excitability by weak direct current stimulation-technical, safety and functional aspects. Supplements to Clinical neurophysiology 56: 255- 276.

30. Nitsche MA, Liebetanz D, Tergau F, Paulus W (2002) [Modulation of cortical excitability by transcranial direct current stimulation]. Nervenarzt 73: 332-335.

31. Nitsche MA, Paulus W (2000) Excitability changes induced in the human motor cortex by weak transcranial direct current stimulation. J Physiol 527: 633-639.

32. Nitsche MA, Paulus W (2001) Sustained excitability elevations induced by transcranial DC motor cortex stimulation in humans. Neurology 57: 1899-1901.

33. Wassermann EM, Grafman J (2005) Recharging cognition with DC brain polarization. Trends Cogn Sci 9: 503-505.

34. LaRowe SD, Myrick H, Hedden S, Mardikian P, Saladin M, et al. (2007) Is cocaine desire reduced by $\mathrm{N}$-acetylcysteine? Am J Psychiatry 164: 1115-1117.

35. Prisciandaro JJ, McRae-Clark AL, Myrick H, Henderson S, Brady KT (2014) Brain activation to cocaine cues and motivation/treatment status. Addict Biol 19: 240-249.

36. Volkow ND, Tomasi D, Wang GJ, Fowler JS, Telang F, et al. (2011) Reduced metabolism in brain "control networks" following cocaine-cues exposure in female cocaine abusers. PLoS One 6: e16573. 
37. Anderer P, Saletu B, Pascual-Marqui RD (2000) Effect of the 5-HT1A partial agonist buspirone on regional brain electrical activity in man: A functional neuroimaging study using low-resolution electromagnetic tomography (LORETA). Psychiatry Res 100: 81-96.

38. Pascual-Marqui RD, Esslen M, Kochi K, Lehmann D (2002) Functional imaging with low-resolution brain electromagnetic tomography (LORETA): A review. Methods Find Exp Clin Pharmacol 24 Suppl C: 91-95.

39. Pascual-Marqui RD, Lehmann D, Koenig T, Kochi K, Merlo MCG, et al. (1999) Low resolution brain electromagnetic tomography (LORETA) functional imaging in acute, neuroleptic-naive, first-episode, productive schizophrenia. Psychiatry Res 90: 169-179.

40. Worrell GA, Lagerlund TD, Sharbrough FW, Brinkmann BH, Busacker NE, et al. (2000) Localization of the epileptic focus by low resolution electromagnetic tomography in patients with a lesion demonstrated by MRI. Brain Topogr 12: 273-282.

41. Nakamura-Palacios EM, de Almeida Benevides MC, da Penha ZagoGomes M, de Oliveira RW, de Vasconcellos VF, et al. (2012) and cognitive changes induced by frontal direct current stimulation in alcoholics according to Lesch alcoholism typology. Int J Neuropsychopharmacol 15: 601-616.

42. Gandiga PC, Hummel FC, Cohen LG (2006) Transcranial DC stimulation (tDCS): A tool for double-blind sham-controlled clinical studies in brain stimulation. Clin Neurophysiol 117: 845-850.

43. da Silva MC, Conti CL, Klauss J, Alves LG, do Nascimento Cavalcante HM, et al. (2013) Behavioral effects of transcranial direct current stimulation (tDCS) induced dorsolateral prefrontal cortex plasticity in alcohol dependence. J Physiol Paris 107: 493-502.

44. Goldstein RZ, Volkow ND (2011b) Oral methylphenidate normalizes cingulate activity and decreases impulsivity in cocaine addiction during an emotionally salient cognitive task. Neuropsychopharmacology 36: 366-367.

45. Goldstein RZ, Volkow ND (2011a) Dysfunction of the prefrontal cortex in addiction: Neuroimaging findings and clinical implications. Nature Reviews Neuroscience 12: 652-669.

46. Volkow ND, Fowler JS, Wang GJ, Telang F, Logan J, et al. (2010) Cognitive control of drug craving inhibits brain reward regions in cocaine abusers. Neuroimage 49: 2536-2543.
47. Boggio PS, Ferrucci R, Rigonatti SP, Covre P, Nitsche M, et al. (2006) Effects of transcranial direct current stimulation on working memory in patients with Parkinson's disease. J Neurol Sci 249: 31-38.

48. Dockery CA, Hueckel-Weng R, Birbaumer N, Plewnia C (2009) Enhancement of planning ability by transcranial direct current stimulation. J Neurosci 29: 7271-7277.

49. Fertonani A, Rosini S, Cotelli M, Rossini PM, Miniussi C (2010) Naming facilitation induced by transcranial direct current stimulation. Behav Brain Res 208: 311-318.

50. Fiori V, Coccia M, Marinelli CV, Vecchi V, Bonifazi S, et al. (2011) Transcranial direct current stimulation improves word retrieval in healthy and nonfluent aphasic subjects. J Cogn Neurosci 23: 2309-2323.

51. Fregni F, Boggio PS, Nitsche M, Bermpohl F, Antal A, et al. (2005) Anodal transcranial direct current stimulation of prefrontal cortex enhances working memory. Exp Brain Res 166: 23-30.

52. Jeon SY, Han SJ (2012) Improvement of the working memory and naming by transcranial direct current stimulation. Ann Rehabil Med 36: 585-595.

53. Jo JM, Kim YH, Ko MH, Ohn SH, Joen B, et al. (2009) Enhancing the working memory of stroke patients using tDCS. Am J Phys Med Rehabil 88: 404-409.

54. Kuo MF, Nitsche MA (2012) Effects of transcranial electrical stimulation on cognition. Clin EEG Neurosci 43: 192-199.

55. Marshall L, Mölle M, Hallschmid M, Born J (2004) Transcranial direct current stimulation during sleep improves declarative memory. J Neurosci 24: 9985-9992.

56. Hajcak G, Anderson BS, Arana A, Borckardt J, Takacs I, et al. (2010) Dorsolateral prefrontal cortex stimulation modulates electrocortical measures of visual attention: evidence from direct bilateral epidural cortical stimulation in treatment-resistant mood disorder. Neuroscience 170: 281-288.

57. Rodrigues LC, Gobira PH, de Oliveira AC, Pelição R, Teixeira AL, et al. (2014) Neuroinflammation as a possible link between cannabinoids and addiction. Acta Neuropsychiatr 26: 334-346.

58. Liao K, Guo ML, Niu F, Yang L, Callen SE, et al. (2016) Cocaine-mediated induction of microglial activation involves the ER stress-TLR2 axis. J Neuroinflammation 13: 33. 